\title{
Assessment of bio-activities of the crude extract and components of Withania somnifera leaves by bioinformatics
}

\author{
Nashi Widodo ${ }^{1}$, Didik Priyandoko ${ }^{3}$, Tetsuro Ishii ${ }^{3}$, Custer Deocaris ${ }^{2}$, Renu Wadhwa ${ }^{2 *}$, Sunil C. Kaul ${ }^{2}$ \\ ${ }^{1}$ Department of Biology, Faculty of Sciences, Brawijaya University, Indonesia \\ ${ }^{2}$ National Institute of Advanced Industrial Science and Technology (AIST), Tsukuba, Ibaraki, Japan \\ ${ }^{3}$ Graduate School of Life and Environmental Sciences, University of Tsukuba, Ibaraki, Japan
}

\begin{abstract}
Traditional herbal medicines are now increasingly being appreciated with Western models of integrative health sciences and evidence-based approach both in the basic research and clinic scenario. Ashwagandha is a commonly used plant in Ayurvedic, Indian traditional medicine. Medicinal value of Ashwagandha (WithaniasomniferaDunal) extends from anti-inflammatory, anti-arthritic, anti-rheumatic, rejuvenation and anti-cancer. Based on the belief that holistic multi-site mechanism of action offers greater chance of success, the traditional Ayurvedicmedicine practices the use of whole herb or its crude extract. It opposes with the mainstream of pharmaceutical industry that uses single and purified molecules. In the present study, we used bioinformatics approach to reveal the mechanism of action of (i) crude extract of Ashwagandha leaf extract and its purified components, (ii) Withanone and (iii) Withaferin A. Whereas p53-p21 was identified as a common signaling pathway for the three kinds of reagents, specific signaling pathways for Withaferin-A and Withanone were identified. Whereas the crude extract and Withanone were selectively toxic to human cancer cells, WithaferinA showed cytotoxicity to the normal cells too. The study suggested that the crude extract or a combinational formulamay be a superior and safenatural reagent for cancer treatment.
\end{abstract}

Keywords: Holistic mechanism, traditional medicine, Withania somnifera

\section{Introduction}

Estimated $80 \%$ of the world population still has no access to modern medicine [2] and rely on traditional home medicine treatments using a variety of herbs. Ashwagandha (Withaniasomnifera) is widely used in Indian natural medicine, Ayurveda that constitutes one of the world's oldest system of hoe medicine. Extracts from different parts of Ashwagandha have been claimed to be valuable for remedies for stress, inflammation, arthritic, antidepressant, immunostimulation, adaptogenic, cardioprotection, rejuvenation, and regeneration $[7,1,3$ $4,8]$. Ayurvedic medical system practices the use of dry powder or crude extract in contrast to the modern medicine that relies on single molecules or pure compound. It is widely believed that the crude extracts may offer ystemic curing

\footnotetext{
*Corresponding address:

Renu Wadhwa

AIST Tsukuba Central 4

1-1-1 Higashi, Tsukuba,

Ibaraki 305-8562, Japan

Email: renu-wadhwa@aist.go.jp

+81-29-861-2521
}

mechanism, multi-site mechanisms of action from complex active compounds and hence provide greater chances for success than a single agent [9]. However, the mechanisms and the multiple target approach lacks laboratory validation. We undertook a bioinformatics study to reveal the mechanism of action of the crude extract and purified components of the Ashwagandha leaves. Aswagandha in comparing gene regulatory circuits in a crude extract preparation vis-à-vis some of its bioactive components.

Initially, gene targets of the crude extract and its purified components were identified by gene silencing approach using human cell culture as a model. In this model, cells were transfecetd with gene silencing small molecules such as siRNA ribozyme and then treated with the crude extract or purified component. Effect of the extractand purified molecules is compared in the control and gene-silenced cells by cell viability assays. By this assay, the genes of which the silencing abrogated the effect of crude extract or the components were selected as candidate gene targets. List of such identified targets was then analyzed by bioinformatics tool "Ingenuity Pathway Analysis" to explore how the identified gene targets interact functionally with each other. We found that crude extract and their purified components act through different pathways. WithaferinA caused 
induction of pathways that signified higher toxicity. In contrast to this, Withanone and the crude extract caused were less toxic suggesting that crude extract may be a safer approach.

\section{Materials and Methods}

\section{Preparation of crude extract from Ashwagandha.}

Ashwagandha ( $W$. somnifera) leaf extracts were prepared as described earlier [5]. The leaves were air dried, ground to a fine powder, and subjected to extraction with methanol $\left(60^{\circ} \mathrm{C}\right)$ in Soxhlet apparatus for 4 to 5 days. The methanol extracts were further extracted with hexane to remove chlorophyll and other pigments and then with diethyl ether that was evaporated to obtain the ether extract. Ether extract solubilized in DMSO was used for the present studies. Whereas, Withanone and Withaferin A have been purchased from Cromadex, USA.

\section{Pathway analysis by gene silencing approach}

shRNAs for several genes as described earlier [14] were cloned in a U6 driven expression vector as described earlier (Wadhwa, et al., 2004). Osteogenic sarcoma (U2OS) cells were plated in 96-well plates, cultured in DMEM (Life Technologies, Gaithersburg, MD), supplemented with $10 \%$ fetal bovine serum in a humidified incubator $\left(37^{\circ} \mathrm{C}\right.$ and $\left.5 \% \mathrm{CO}_{2}\right)$. Cells $(\sim 50-60 \%$ confluency) were transfected with $50 \mathrm{ng}$ of the plasmid DNA. After $48 \mathrm{~h}$, cells were treated with crude extract $(6 \mathrm{lg} / \mathrm{ml})$, withaferin $\mathrm{A}(1.5 \mathrm{lM})$ and Withanone $(25 \mathrm{lg} / \mathrm{ml})$. Cell viability was measured by Alamar BlueTM assay (BioSourceInterna- tional, USA). Empty shRNA vector was used as a control. The shRNAs that abrogated the killing effect of i-Extract and its fractions were considered as involved in their effect and were analyzed for pathways by Ingenuity Pathways Analysis software (www.ingenuity.com).

\section{Results and Discussion}

Function of candidate target gene and the possible mechanism of toxicity of crude and its fractions were analyzed by Ingenuity Pathways Analysis (Ingenuity System, www.ingenuity.com) (Figure 1). The gene silencing based citotoxicity analysis revealed that the crude Extract and its components have different pattern in killing mechanism of cancer cell (Figure 2).

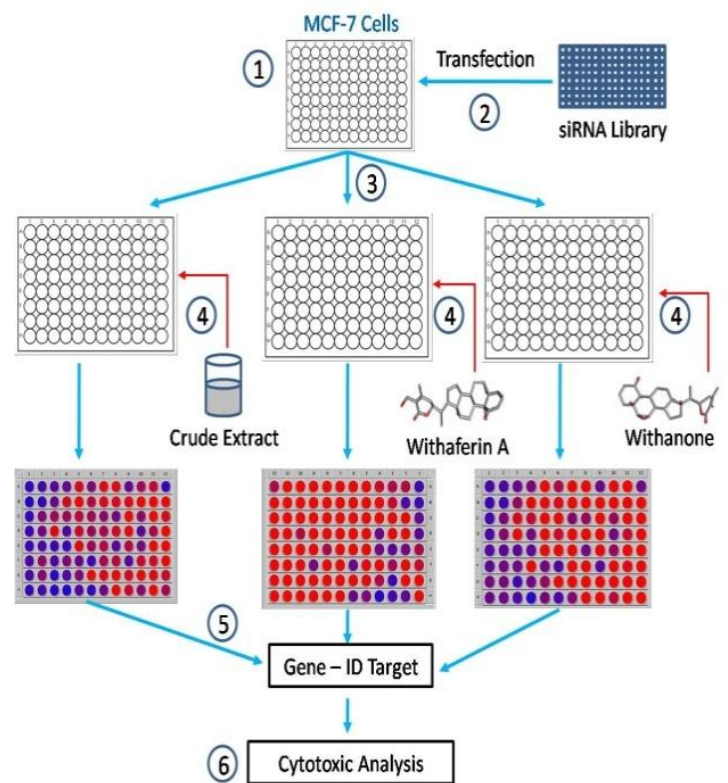

Figure 1. Diagram off functional genomic assays were employing small hairpin RNA (shRNA; siRNA Library) to identified gene targets of crude extract of Ashwagandha, withanone and withaferin A. MCF-7 were grown in 96 well plate (1) for $24 \mathrm{~h}$, then were transfected with siRNA library(2). After $12 \mathrm{~h}$, cells were splite into 3 plate (3) and treated with the respective drugs (4). After $24 \mathrm{~h}$ exposure to the crude extracts, gene targets were identified based on dead (Blue) or live cell (red) (5). The target genes been used to performed cytotocitiy analysis using IPA software.

Taken together, the data revealed that crude extract kill cancer cells through 3 pathways: cell cycle G2/M, Oxidative stress and p53 Signaling. In addition to these pathways, Withanone appeared to cause induction of Apoptosis, and Oxidative stress signaling. Withaferin $\mathrm{A}$ also caused induction of NF-kappaB that was not significant in the Withanone treated cells.

Interestingly, whereas crude extract and its compounds seemed to affect in different pathways, p53 signaling was identified as one of the common pathways. We examined p53 activity in the cells and found that infact p53 was activated by treatment of cells with crude extracts well as the pure components. Mechanism of such activation was also attempted. The activity of wild-type p53 is regulated by multiple factors, including its interactions with binding partners.

In transformed cells, mortalin, a hsp70 family member, interacts and inactivates p53 by sequestering it in the cytoplasm [6]. We found that in cells treated with crude extract and its compnents, p53-mortalin complexes are abrogated and there is a significant increasein the nuclear translocation of p53 and shift of mortalin staining from the perinuclear to pancytoplasmicpattern (Figure 3). 

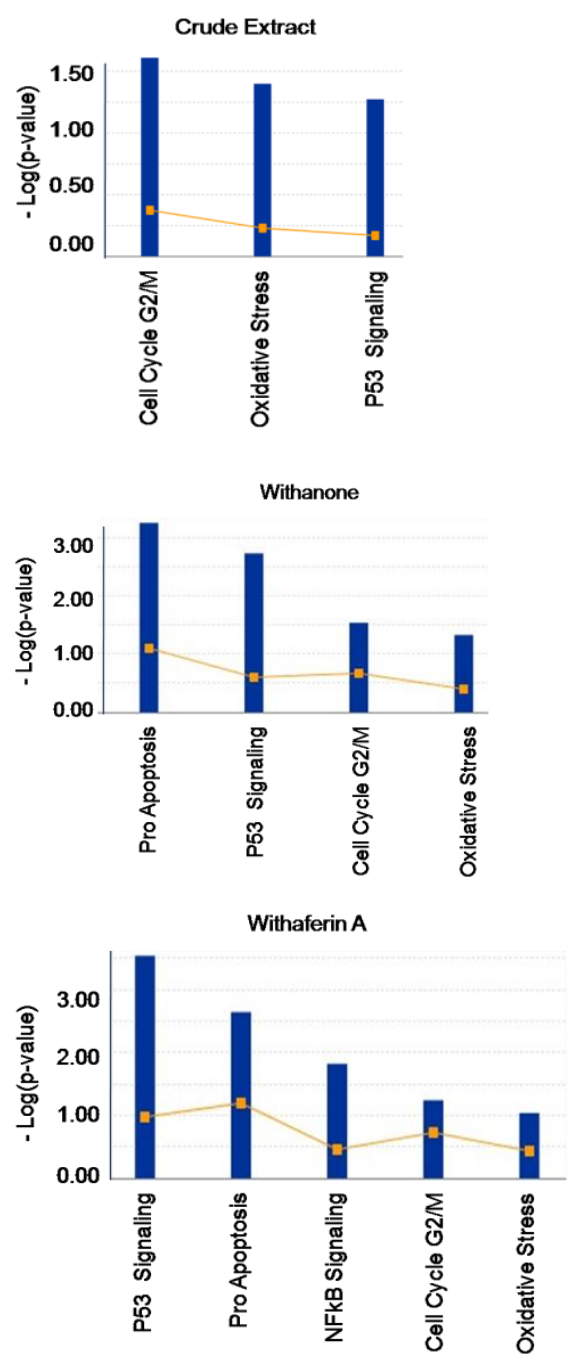

Figure 2. Cytotoxicity Mechanism Comparisons of crude extracts, Withanone and WithaferinA. Crude extract showed greater selectivity than others, which is statistically impacted on only three pathways, whereas the effects of Withanone and WithaferineA were more spread.

Such shift in thestaining pattern of mortalin and increase in $\mathrm{p} 53$ has earlier been demonstrated to be associated with the induction of senescence in cancer cells [11,12]. Based on these findings, we concluded that the three kind of compounds cause senescence in cancer cells. These findings suggested that crude extract of Ashwagandha, WithaferinA, Withanonedisrupt mortalin-p53 interactions and thus act as anti-mortalin molecules resulting in reactivation of $\mathrm{p} 53$ function.

We next tested the effect of three components on normal human cells by the similar approach. As shown in Figure 4, we found that whereas the crude extract and Withanone were nontoxic to normal cells, Withaferin A was toxic [15]. It caused activation of p53 in normal cells that was associated with the acticvation of its downstream regulator p21.
Both the crude extractand Withanone did not cause induction of p53 or p21 in normal cells.

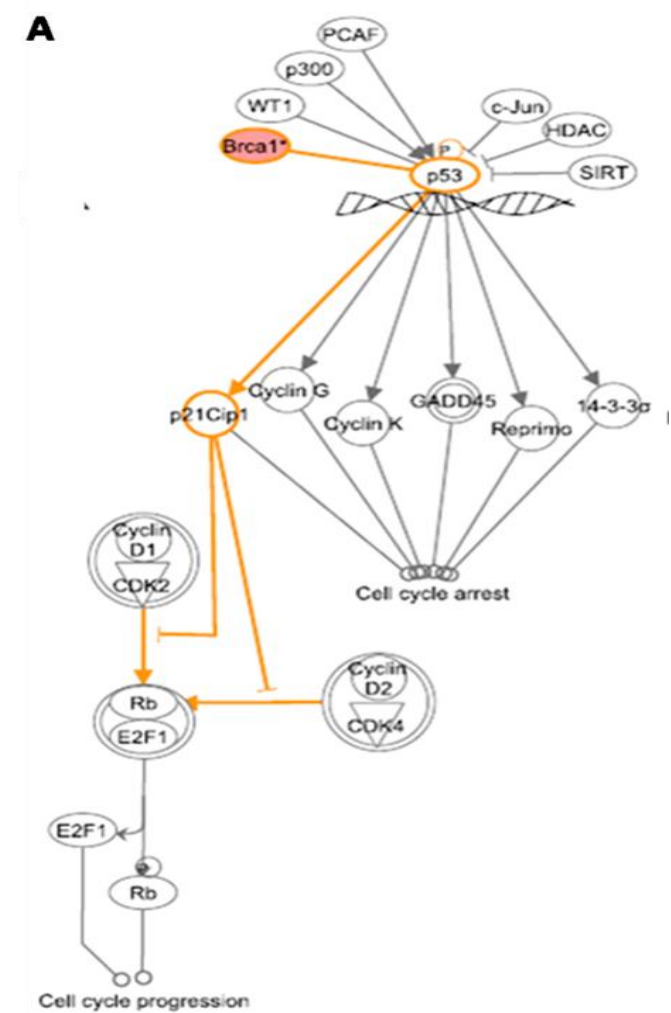

B
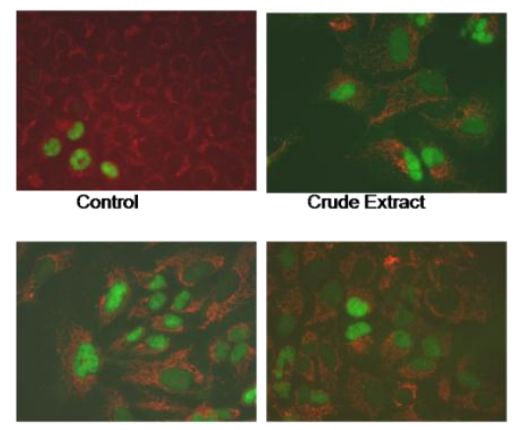

Withaferin A

Withanone

C

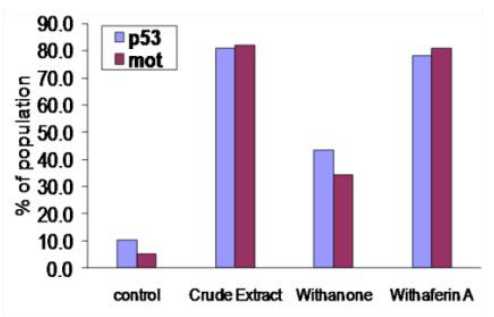

Figure 3. Common pathways targeted by crude extract, withaferinAdanWithanone. It showed that the BRCA1 is key target gene to stop cell cycle progession (A) trough activation of p53 function (C) and caused a shift in the staining pattern of mortalin (red) from perinuclear to pancytoplasmic), p53 was translocated into the nucleus of cancer cells (green nuclear staining) (B).

In fact, p21 was seen as down-regulated suggested that such suppression of p53-p21 in 
normal cells may cause their rejuvenation. We tested this by a variety of molecular markers and lifespan studies and found that indeed the cells cultured in Withanone-supplemented medium have lower degree of molecular damage and are protected against the oxidative damage $[14,15]$. The cells cultured in Withanone supplemented medium showed lifespanextension suggesting that Withanone is a candidate anti-aging reagent.
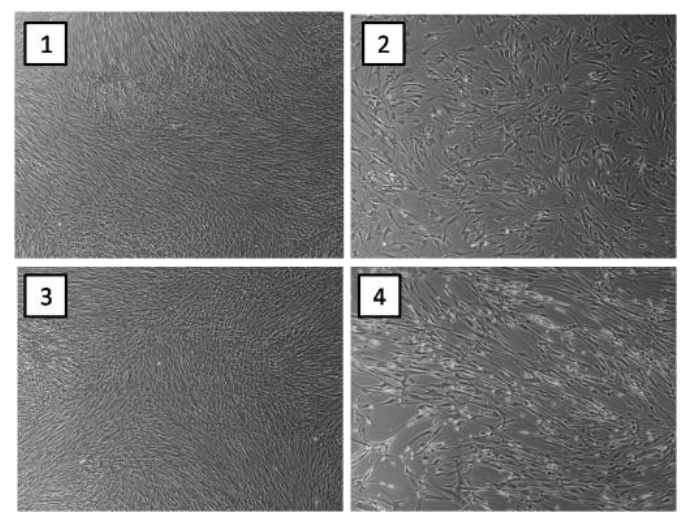

Figure 4. Withaferin have more toxicity activity in normal cell (TIG-1) (4) then withanone (3), Crude extract (2) and control (1).

By using a combined gene-silencing and bioinformatics approach, we have found that the crude extract and its components differ in their bioactivity and the mechanism of action. Whereas Withaferin A is toxic to normal cells, thecrude extract did not cause toxicity suggesting that Withanone is natural antidote of Withaferin A.

\section{Conclusion}

Ashwaganda ( $W$. somnifera)leaf extract kills cancer cells selectively. Its components, Withaferin $\mathrm{A}$ and Withanone have different gene targets and mechanism of action. Whereas Withaferin A caused toxicity to normal cells, Withanone was safe and hence can be recruited in safe cancer therapeutics.Furthermore, the combinational approach for Withaferin A and Withanone for anticancer, antiaging effects for cancer and normal cells, respectively is warranted.

\section{Reference}

[1] Archana R and Namasivayam A. 1999. Antistressor effect of Withaniasomnifera. J Ethnopharmacol. 64:91-3.

[2] Hegde BM.2003. Future Medicare system. J Assoc Physicians India.51:606-608
[3] Davis L and Kuttan G. 2002. Effect of Withaniasomnifera on cell mediated immune responses in mice. J Exp Clin Cancer Res. 21:585-90.

[4] Davis L and Kuttan G. 2002. Effect of Withaniasomnifera on CTL activity. J Exp Clin Cancer Res. 21:115-8.

[5] Kaur K, Rani G, Widodo N, Wadhwa R. 2004. Evaluation of the anti-proliferative and anti-oxidative activities of leaf extract from in vivo and in vitro raised ashwagandha. Food ChemToxicol. 42:2015-20

[6] Mihara M, Erster S, Zaika A. 2003. p53 has a direct apoptogenic role at the mitochondria. Mol Cell. 11:577-90

[7] Scartezzini P and Speroni E. 2000. Review on some plants of Indian traditional medicine with antioxidant activity. J Ethnopharmacol. 71:23-43.

[8] Singh B, Chandan BK and Gupta DK. 2003. Adaptogenic activity of a novel withanolide-free aqueous fraction from the roots of Withaniasomnifera Dun. (part II). Phytother Res. 17:531-6.

[9] Patwardhan B. 2005. Ethnopharmacology and drug discovery. J Ethnopharmacol.100:50-52

[10] Wadhwa R, Yaguchi T, Kaur K, Kaul S. 2004. Use of a randomized hybrid ribozyme library for identification of genes involved in muscle differentiation. J Biol Chem. 279:51622-9.

[11] Wadhwa R, Sugihara T, Yoshida A, Kaul S. 2000. Selective toxicity of MKT-077 to cancer cells is mediated by its binding to the hsp70 family protein mot-2 and reactivation of p53 function. Cancer Res. 60:6818-21.

[12] Wadhwa R, Kaul SC, Mitsui Y, Sugimoto Y. 1993.Differential subcellular distribution of mortalin in mortal and immortal mouse and human fibroblasts. Exp Cell Res. 442-8.

[13] Wermuth CG. 2004. ultitargeted drugs: the end of the "onetarget-one-disease" philosophy?.Drug Discov Today.9:826-827.

[14] Widodo, N., Y. Takagi, sheresta, BG, Ishii T, Wadhwa and R, Kaul S. 2008. Selective killing of cancer cells by leaf extract of Ashwagandha: Components, activity and pathway analyses. Cancer Lett. 262: 37-47.

[15] Widodo N, Kaur K, Shrestha BG, Takagi Y, Ishii T, Wadhwa R, Kaul SC. 2007. Selective killing of cancer cells by leaf extract of Ashwagandha: identification of a tumor-inhibitory factor and the first molecular insights to its effect. Clin Cancer Res. 1;13(7):2298-306 